REVISTA ECONOMÍA

Vol. 7I, N. ${ }^{\circ}$ II4 (noviembre 20I9), 73-87

\title{
LA METAMORFOSIS DEL DESARROLLO ECONÓMICO
}

\author{
THE METAMORPHOSIS OF ECONOMIC DEVELOPMENT
}

BRYAN PÉREZ ALMEIDA

Flacso-Ecuador

Recepción manuscrito: 3ode noviembre de 2019

Aceptación versión final: 14 de octubre de 2019

\begin{abstract}
RESUMEN Quizá la mayor preocupación en las naciones es el crecimiento económico, más aún cuando son países subdesarrollados. Para lo cual, las escuelas del desarrollo económico han elaborado varias teorías a fin de que los países menos afortunados alcancen a los países desarrollados. Sin embargo, la fragmentación del mundo es imperante y, en el sistema capitalista, se puede encontrar naciones atoradas en el estancamiento económico, mientras sueñan en el desarrollo económico. Lo cual surge en el período de 2000 a 2017 en un pool de 122 países, donde muchos quedan colapsados en el estancamiento económico.
\end{abstract}

palabraS ClaVe Desarrollo económico, fragmentación, estancamiento económico.

ABSTRACT Perhaps the greatest concern in nations is economic growth, even more so when they are underdeveloped countries. For which, schools of economic development have developed several theories so that the less fortunate countries reach the developed countries. However, the fragmentation of the world is prevalent in the capitalist system. So we can find nations stuck in economic stagnation while they dreaming of economic development. This emerges in the period from 2000 to 2017 in a pool of 122 countries, where many collapse into economic stagnation.

KEYWORDS Economic development, fragmentation, economic stagnation.

JEL CODES C10, F43, O11.

\section{INTRODUCCIÓN}

Una de las principales preocupaciones de los líderes en cada nación es el crecimiento económico del país que gobiernan. Más aún cuando son representantes de países que difícilmente han logrado la metamorfosis del subdesarrollo al desarrollo económico, ${ }^{1} \mathrm{y}$ en su lugar han quedado estancados en el atraso económico, donde los patrones entre países subdesarrollados y desarrollados parecieran endémicos, ${ }^{2}$ al punto que han sido clasificados en dos grandes grupos que, a su vez, pueden ser identificados como centro y periferia en el pensamiento estructuralista, o metrópoli y satélites en la corriente dependentista, e incluso, por el 
nivel de ingreso percápita, ${ }^{3}$ como países de ingreso bajo, ingreso mediano bajo, ingreso mediano alto e ingreso alto.

Sin embargo, se podría pensar que la división del mundo en dos o cuatro grandes grupos obedece a la lógica del sistema capitalista, que alinea y envuelve a las naciones a nivel mundial en la misma estructura de acumulación en la que se encuentran, interactúan y relacionan, vía comercio internacional, productores especializados en materias primas y bienes con escaso valor agregado frente a productores de manufacturas con elevado valor agregado donde estos últimos obtienen un mayor beneficio con respecto a los productores de materias primas (Gunder-Frank, 1969; Ruy Marini, 1973; Pushan Dutt, Mitra y Rajan, 2009), lo que puede ser producto de: (1) el deterioro de los términos de intercambio (Prebisch, 1950; Baer, 1963; Ocampo, 2001) donde el desarrollo de unos está en función del subdesarrollo de otros (Emmanuel, 1972; Amin et al., 1976), o, visto desde una perspectiva menos estructuralista y/o dependentista, es ocasionado por (2) la insuficiente inversión para generar un proceso de industrialización donde la producción de materias primas y alimentos superen las endebles elasticidades ingreso de importación (Thirlwall, 1983; Sarkar, 1997), o porque (3) la etapa del subdesarrollo al desarrollo no ha sido superada (Rostow, 1963).

No obstante, las relaciones asimétricas que ciñen los países desarrollados y subdesarrollados en una espiral de competencia, intercambio desigual (Prebisch, 1950) y dotaciones industriales desequilibradas (haciendo referencia a la ley de Verdoorn en Dixon y Thirlwall, 1975), arrojan como resultado esta fisión del mundo que, al parecer, trasciende la geografía, cultura o clima (Acemoglu y Robinson, 2014), pero que acata a un sistema capitalista donde la concentración, exportación de capitales y búsqueda de ascendentes ganancias a costa de una mayor explotación laboral en tierras ajenas, permite pensar en un sistema-mundo como el de Wallrestein (2005), donde el camino por recorrer de países subdesarrollados para llegar a ser desarrollados, es un torrente, en cuya base se encuentra un ser con piernas de barro, que camina, pero difícilmente hacia la cima.

De modo que surge la presunción que en el sistema capitalista coexisten al menos dos grupos, desarrollados y subdesarrollados, que participan en el mercado mundial en búsqueda del crecimiento económico prometido por las economías desarrolladas (Baran, 1950), pero que más bien, ocurre lo contrario y quedan relegadas en el atraso económico; donde el big push y el take-off (Snowdown, 2009; Krugman, 1995; Murphy, Shleifer y Vishny, 1989; Easterly, 2006) poco o nada han podido hacer para que las economías subdesarrolladas se alejen de un equilibrio de estado estacionario de bajo ingreso a un equilibrio de alto ingreso (Barro y Sala-iMartin, 1995); arrojando como evidencia la no convergencia de los países hacia un mismo estado estacionario, es decir, países más «desarrollados» que otros (Quah, 1996; Baddeley, 2006).

Este resultado llama la atención, ya que dentro de la economía existe una ramificación dedicada al estudio del desarrollo económico, sobre todo en el período post de la segunda Guerra Mundial (Meier, 2001), sin embargo, las reflexiones sobre el desarrollo económico - excepto por la corriente dependentista y estructuralista - están en el seno de los países desarrollados cuyo contexto es ajeno a la de naciones subdesarrolladas (Baran, 1950; Palma, 1978). Por lo tanto, el fracaso de los recetarios para el desarrollo económico en países subdesarrollados, se debe en parte, a la aceptación de teorías desarrolladas por países desarrollados, así como a la 
corrupción (Easterly, 2006), mala utilización de los recursos (Botta, 2010), fallas de Gobierno (Krueger, 1990) y un escaso desarrollo cultural — sobre todo en aquellos países que fueron colonia- (Acemoglu y Robinson, 2014).

Por el anterior esbozo sobre el desarrollo económico y, al parecer, su inefectividad para dejar atrás el atraso económico en las naciones menos favorecidas, la presente reflexión gravitará alrededor del estancamiento económico y las corrientes del desarrollo, pues éstas idean una senda hacia un mismo estado estacionario - todos los países llegan a un mismo equilibrio de alto ingreso-, pero más bien surgen varios estados estacionarios, donde pocos países llegan a un equilibrio de alto ingreso, y muchos quedan relegados en equilibrios de bajo ingreso. Para dar evidencia no solo a nivel teórico de una fragmentación del mundo bajo las generalidades como desarrollado y subdesarrollado, centro y periferia, metrópoli y satélite; sino también, empíricamente, el presente artículo propone el desarrollo de un análisis de clustering, ya que es una herramienta capaz de formar conglomerados según las similitudes con base en indicadores de desarrollo económico, apertura comercial y estructura productiva de los países; arrojando una o varias aglomeraciones, donde se podrá identificar un solo estado estacionario (convergencia), múltiples estados estacionarios (divergencia), así como los twin picks en Quah (1996). El conjunto de datos para tal propósito ha sido recolectado en el período 2000 a 2017 en un total de 122 países con información sobre desarrollo económico, donde los resultados señalan que en casi dos décadas del siglo xxi muchos países solo han podido soñar con el desarrollo económico pues el estancamiento no ha sido superado.

De este modo, la metodología de clustering al permitir la formación de conglomerados según las similitudes en materia de desarrollo económico, apertura comercial y estructura productiva, será el instrumento que buscará dar respuesta a la siguiente interrogante: ¿El desarrollo económico es posible para todos los países, o solo para una proporción de ellos? Los resultados muestran evidencia análoga a los clubs de la convergencia (Chatterji, 1992; Quah, 1996; Ben-David, 1998) reportada en la literatura, solo que, en un nivel más profundo pues se forman varias agrupaciones, donde unos pocos países se mantienen - a lo largo del tiempoen los clústeres más favorecidos, mientras la mayoría no ha trascendido los clústeres de menor dinamismo. No obstante, los resultados del presente artículo no solo coinciden con los autores anteriores cuyas metodologías de estimación son diferentes a la actual, también se armonizan con el estudio de Brida, Garrido y Muredu (2014), enfocados en la convergencia de las regiones italianas; y los análisis de Monfort, Cuestas y Ordóñez (2013); Borsi y Metiu (2015) y Lyncker y Thoennessen $(2016)^{4}$ sobre el proceso de convergencia en la Unión Europea.

En realidad, hasta mi entendimiento en la literatura, el trabajo de Brida, Garrido y Muredu (2014) es el único que utiliza un análisis de clustering para identificar el proceso de convergencia en las regiones italianas; más no los estudios de Monfort, Cuestas y Ordóñez (2013); Borsi y Metiu (2015) y Lyncker y Thoennessen (2016), pues si bien aplican un enfoque de clustering, utilizan el algoritmo diseñado por Phillips y Sul (2007) para determinar clubs en la Unión Europea. Sin embargo, la propuesta actual pretende alcances de mayor escala por el número de países, que es mayor, y el método de clustering, representando, así, una novedad a la vez que un desafío. Pues si bien el proceso de convergencia ha sido estudiado con otras metodologías como series temporales en panel de datos (Evans, 1998; McCoskey, 2010), modelos teóricos 
(Barro y Sala-i-Martin, 1995), árboles de regresión (Postiglione, Benedetti y Lafratta, 2010) o medidas de entropía (De Siano y D’Uva, 2006; Maasoumi, Racine y Stengos, 2007; Frey, Frey y Wieslhuber, 2013), los resultados en la actual reflexión —oriundos de una metodología relativamente nueva- parecieran ser relevantes y un aporte a la literatura existente. De este modo, tras elaborar una introducción que encuadre el tópico en estudio y sus alcances, el resto del artículo se compone de las siguientes secciones. La segunda sección abordará la revisión de literatura sobre artículos relacionados a la actual propuesta; el tercer apartado detallará los datos y la metodología a utilizar; y a posterior finalizar con la sección de principales resultados y conclusiones de la propuesta actual.

\section{REVISIÓN DE LA LITERATURA}

Como se mencionó anteriormente, la literatura sobre el desarrollo económico cuenta con investigaciones teóricas, empíricas de series de tiempo, panel de datos, procesos estocásticos y unos cuantos, con métodos de agrupación, aunque con un pool de datos menor al actual. En ese sentido, la técnica de clústeres para identificar la senda del desarrollo (metamorfosis) que han tenido los países desde 2000 a 2017, parece novedosa por la segmentación de grupos a través del cálculo de distancias entre países, a fin de apilar los más homogéneos dentro de un mismo grupo. Es decir, aquellas naciones que no han acelerado su crecimiento económico y/o pib per cápita, se encontrarán en un grupo inferior a aquellos que sí lo han conseguido e, incluso, se encontrarán alejados de países que evidenciaron un crecimiento económico más vertiginoso. Sin embargo, es necesario reseñar ciertas investigaciones que han sido relevantes en la literatura del crecimiento y desarrollo económico.

Así, se parte con el modelo de crecimiento exógeno de Solow y Swan (1956) donde los autores señalan que el crecimiento económico es posible a través del incremento en el ahorro con el fin de invertir en capital y, así, lograr una retroalimentación entre las variables mencionadas que permita alcanzar un estado estacionario de ingreso alto — crecimiento económicoNo obstante, el modelo de crecimiento exógeno de Solow y Swan (1956) sería retomado más adelante por Barro y Sala-i-Martin (1995), quienes pretenden explicar las trampas de pobreza que representan una barrera para los países menos desarrollados, pues quedan colapsados en estados estacionarios de bajo ingreso, mientras otros países se encuentran enraizados en estados estacionarios de alto ingreso - clubes de la convergencia-. Los autores determinan la existencia de dos equilibrios estables, de bajo y alto ingreso, y uno inestable que es de transición entre los equilibrios de bajo y alto ingreso que para despegar del equilibrio de bajo ingreso, superando las fuerzas gravitatorias ${ }^{5}$ del equilibrio inestable y llegar al equilibrio de alto ingreso, es necesario un aumento de la inversión en capital y disminución del crecimiento poblacional.

Por lo tanto, en la contribución de Barro y Sala-i-Martin (1995) se puede identificar que los países llegan a distintos estados estacionarios, es decir, no convergen al mismo equilibrio. El cual representaría un hallazgo desarrollado por varios autores en la literatura del crecimiento económico y los clubes de la convergencia. Por ejemplo, Chatterji (1992) testea la hipótesis de los clubes de la convergencia a nivel teórico y empírico. El autor toma la base de datos de Heston-Summers (1988) sobre el ingreso per cápita de los países en el periodo de 1960 a 1985 y 
comprueba que los países no convergen hacia un mismo estado estacionario, más bien, hacia estados estacionarios relativos: de renta baja y renta alta. Asimismo, Quah (1996) realiza un estudio donde encuentra que la distribución del ingreso de más de 120 países que inicia como unimodal, llega a ser bimodal, formando dos picos, uno de países ricos y otro de países pobres, rebelando así que al pasar de un tiempo $t$ a un tiempo $t+s$ la convergencia hacia un solo equilibrio de estado estacionario no se ha dado y, más bien, la convergencia surge hacia un equilibrio de bajo y alto ingreso.

Por su parte, Evans (1998) utilizando el conjunto de datos de Maddison (1991) y la Penn World Table de 1991, establece un panel de datos con 54 países en el periodo de 1950 a 1990. Donde el desempeño económico de los 13 países más educados de los 27 más ricos, parece ser explicado por las teorías de crecimiento exógenas; mientras que el desempeño económico de los 27 países más pobres no parece ser bien explicado por las teorías de crecimiento endógenas, lo que se debe a la interrumpida difusión tecnológica entre países ricos y pobres, lo que a su vez acarrea el fenómeno de la divergencia entre estos. De igual modo que la convergencia relativa, pero ahora analizada ulteriormente en el África subsahariana, McCoskey (2002) elabora un panel de datos en el período de 1960 a 1969 para los países del África subsahariana con información de la Penn World Table 1991. El autor extrajo información sobre el gasto gubernamental, apertura de la economía, capital por trabajador, PIB per cápita real de la economía, pib real por trabajador y nivel de vida, cuyos resultados señalan que la evidencia no es suficiente para hablar de una convergencia en el nivel de vida en el África subsahariana, más bien, se forman clubes de la convergencia, pero en niveles inferiores.

En la misma línea de resultados, el trabajo realizado por Easterly (2006) recopila literatura donde la convergencia entre países no ha sido lograda, incluso con grandes cantidades de capital humano; y a partir de esto, el autor establece con información del ingreso per-cápita, un pool de 137 países en el período de 1950 a 2001, que, de acuerdo al ingreso per cápita divide en quintiles al pool de países. Los resultados hallados indican que los dos quintiles superiores experimentaron el mayor crecimiento en el pool de datos, mientras que los de peor desempeño fueron los del quintil intermedio; asimismo, determinó que la ayuda extranjera en los países del segundo quintil, no estimuló su crecimiento económico, más bien lo disminuyó, a la vez que los países del primer quintil evidenciaron un mejor desempeño económico pese a no recibir ayuda extranjera. Sin embargo, el desempeño económico positivo que exhibieron no fue tan significativo para llegar a formar parte de los dos primeros quintiles, es decir, los países convergieron a distintos equilibrios — fenómeno de la divergencia-.

En correspondencia a los anteriores autores, Baddeley (2006) analiza los efectos de la globalización en el proceso de convergencia o divergencia de los países. Si bien los países menos desarrollados han tenido un mayor acceso al mercado internacional, flujos de capitales e información - con la introducción del internet - en la globalización, también existen efectos negativos, pues la evidencia empírica sugiere que no se ha suscitado un proceso de convergencia, sino, más bien, de divergencia, siendo más aguda en los países menos desarrollados donde los niveles de desigualdad han ido en aumento.

Por otra parte, y con una metodología diferente a las usuales para el análisis de la convergencia, Frey, Frey y Weislhuber (2013) desarrollan un análisis de árbol de regresión para 
determinar la convergencia en 14 regiones de Kazajistán en el período de 1997 a 2008. Los autores determinan que el origen de los clubes de la convergencia se encuentra asociado a la dotación exógena de recursos naturales y no la dependencia hacia los mismos. Es decir, la disponibilidad inicial de recursos causa los clubes de la convergencia.

Mientras tanto, a nivel de región, Borsi y Metiu (2015) estudian por medio de hechos estilizados y análisis de clúster la convergencia en los Estados miembros de la Unión Europea en el período de 1970 a 2010. Los resultados sugieren la existencia de clubes de la convergencia, a causa de la geografía, pues existe una brecha creciente desde 1990 entre el sudeste - los grupos de mayor dinamismo económico- y nordeste de Europa - grupos cuyo desempeño se encuentra por debajo de la media de la UE-; así como por la pertenencia o no a la Unión Europea. Asimismo, pero ahora para Italia, donde Brida, Garrido y Muredu (2014) analizan la evolución de los niveles de renta en 20 regiones en el período de 1970 a 2004 a través de un análisis de clustering, los autores determinan un club de la convergencia centro-norte de alto rendimiento en relación al club de bajo rendimiento sur-islas. Es decir, existen clubes que exhiben un desempeño asimétrico, que en parte responde —o se relaciona - con la geografía italiana, tal como sucede en el estudio realizado por Brida, Garrido y Muredu (2014).

Ahora bien, la literatura existente sobre procesos de convergencia, clubs de la convergencia y fenómenos de la divergencia es vasta, de hecho, abarcarla en su totalidad excede los límites del presente trabajo. Por ello, solo se ha reseñado una fracción de la literatura que muestra cómo las teorías del desarrollo enfocadas en el aumento del PIB per cápita no ha podido conseguir la convergencia entre países, de hecho, podrían explicar más bien lo contrario, pues en lugar de coincidir en un equilibrio de alto ingreso, se ha formado múltiples equilibrios, donde los países menos desarrollados son atraídos hacia los equilibrios de bajo ingreso donde difícilmente pueden escapar, pues la dinámica comercial asimétrica entre productores de manufacturas y materias primas, el deterioro de los términos de intercambio, la escasa industrialización, tecnología, bajos niveles de capital humano y las teorías propagadas por los países desarrollados - entre muchos otros factores - han provocado una fragmentación del mundo que no ha sido mitigada por ninguna de las escuelas del pensamiento económico. Lo que, a su vez, representa un reto, sobre todo para los países subdesarrollados, pues son ellos quienes deben reunir a los grupos pertinentes y planear nuevas estrategias de crecimiento económico acorde a su realidad y despojarse - al menos la mayoría - de las teorías de los países desarrollados.

\section{DATOS Y MÉTODO DE AGRUPAMIENTO}

A continuación, se detallará la información y el método de agrupamiento que permitirá identificar grupos de países con una dinámica de crecimiento más vertiginosa que otros. Dicha información será extraída de la Penn World Table 91 y del Banco Mundial, donde se pueden encontrar características a nivel país sobre productividad, comercio, trabajo, demográfica y financieras, que son relevantes en la agenda del desarrollo económico (Todaro y Smith, 2012; Romaguera, 1992). Sin embargo, la disponibilidad de información ha sido un obstáculo en la elaboración de la base de datos, pues a priori se esperaba que la información sea lo más antigua posible, y al encontrarse disponible solo para una porción de los países y en pocas variables 
Tabla 1: Selección óptima de conglomerados

\begin{tabular}{|c|c|c|c|c|c|c|c|c|c|}
\hline \multirow[b]{2}{*}{ Año } & \multirow[b]{2}{*}{$\mathrm{kl}$} & \multirow[b]{2}{*}{ hartigan } & \multicolumn{6}{|c|}{ Indicadores para una óptima selección de clústers } & \multirow[b]{2}{*}{ Cluster óptimo } \\
\hline & & & cindex & silhouette & ball & ratkowsky & ptbiserial & mcclain & \\
\hline 2000 & 5 & 5 & 2 & 5 & 3 & 5 & 4 & 5 & 5 \\
\hline 2001 & 10 & 5 & 2 & 5 & 5 & 5 & 4 & 5 & 5 \\
\hline 2002 & 5 & 5 & 2 & 5 & 3 & 5 & 4 & 5 & 5 \\
\hline 2003 & 5 & 5 & 2 & 5 & 3 & 5 & 5 & 5 & 5 \\
\hline 2004 & 5 & 5 & 2 & 5 & 3 & 4 & 5 & 5 & 5 \\
\hline 2005 & 5 & 5 & 2 & 5 & 3 & 5 & 5 & 5 & 5 \\
\hline 2006 & 10 & 5 & 5 & 5 & 3 & 5 & 6 & 5 & 5 \\
\hline 2007 & 5 & 6 & 5 & 2 & 5 & 5 & 5 & 2 & 5 \\
\hline 2008 & 5 & 5 & 6 & 2 & 3 & 5 & 5 & 2 & 5 \\
\hline 2009 & 4 & 5 & 2 & 5 & 3 & 5 & 5 & 4 & 5 \\
\hline 2010 & 5 & 4 & 6 & 5 & 3 & 6 & 5 & 5 & 5 \\
\hline 2011 & 5 & 5 & 2 & 5 & 3 & 5 & 5 & 4 & 5 \\
\hline 2012 & 5 & 5 & 10 & 2 & 3 & 5 & 5 & 2 & 5 \\
\hline 2013 & 5 & 3 & 10 & 2 & 3 & 5 & 5 & 5 & 5 \\
\hline 2014 & 6 & 5 & 8 & 5 & 3 & 5 & 6 & 5 & 5 \\
\hline 2015 & 5 & 5 & 7 & 5 & 3 & 5 & 5 & 5 & 5 \\
\hline 2016 & 5 & 5 & 7 & 5 & 3 & 4 & 5 & 5 & 5 \\
\hline 2017 & 5 & 5 & 6 & 5 & 3 & 5 & 5 & 6 & 5 \\
\hline
\end{tabular}

Elaboración: propia.

- PIB, PIB per cápita y esperanza de vida - el conjunto de datos ha sido reducido al período de 2000-2017 donde la mayoría de países y variables cuentan con información o, al menos, el porcentaje de datos perdidos no supera el 10\%.

De este modo las variables recolectadas en el período de 2000 a 2017 en los 122 países, fueron seleccionadas de acuerdo a la literatura. En Solow y Swan (1956), se resalta la importancia del stock de capital, la inversión - representada la FBKF con respecto al PIB-y tasa de crecimiento real de la economía. Dixon y Thirlwall (1975) destaca el nivel de precios y la producción manufacturera - representada por ratio del valor agregado de la industria con respecto al PIB nominal-. Sarkar (1997) y Bota (2010) toman al tipo de cambio - representado con el tipo de cambio nominal con respecto al dólar de EE. UU.-, la proporción de las exportaciones de mercancías con respecto al PIB, la proporción de las importaciones de mercancías con respecto al PIB y la estructura de la balanza comercial - representada con ratio de exportaciones menos importaciones con respecto al PIB nominal-como relevantes. En Romaguera (2012) por su parte menciona la importancia del crédito, gasto e ingresos fiscales - representados por el crédito al sector público no financiero más el crédito al sector privado con respecto al 
PIB nominal, ingresos fiscales con respecto al PIB nominal y gastos menos ingresos fiscales con respecto al PIB nominal-. Ahora bien, según Todaro y Smith (2012), así como en Monfort, Cuestas y Ordóñez (2013) es importante variables de estructura que permitan entender el proceso de desarrollo económico, por lo cual también utilizaremos proporción de personas contratadas, proporción de personas desempleadas y esperanza de vida.

Con las variables mencionadas y la metodología de segmentación de clustering, se pretende determinar en el pool de 122 países varios grupos que permitan identificar la dinámica de crecimiento o estancamiento económico que han evidenciado en las casi dos décadas del siglo Xxi. Para lograr lo anterior, el ejercicio de agrupamiento y construcción de clústeres se realizará en el software de programación R, empleando la función hclust del paquete stats. No obstante, si la pérdida de información hubiera sido nula, el paquete $\mathrm{NbClust} \mathrm{habría} \mathrm{sido} \mathrm{utilizado,}$ puesto que identifica los grupos - dado un método de disimilitud y agrupamiento- sin la necesidad de especificar el número de clústeres, es decir, se deja que los datos hablen. Sin embargo, no fue posible y, por ello, se ha realizado la selección del número de conglomerados o clústeres de acuerdo a los indicadores $k l$ (Krzanowski y Lai, 1988), hartigan (Hartigan, 1975), cindex (Hubert y Levin, 1976), silhouette (Rousseeuw, 1987), ball (Ball y Hall, 1965), Ratkowsky (Ratkowsky y Lance, 1978), ptbiserial (Milligan, 1980) y mcclain (McClain y Rao, 1975). ${ }^{6}$ Los cuales buscan determinar el número óptimo de conglomerados, pero también, maximizar la explicación del conjunto de datos a través de las similitudes en los clústeres. No obstante, los resultados detallados en la tabla 1 (ver Tabla 1 ) sobre el número óptimo de conglomerados señalan una volatilidad, pues según los criterios de validación (indicadores) en un mismo año, el número clústeres a elegir es diferente. Por lo cual, se decidió elegir el número de clúster con mayor frecuencia entre los índices de selección.

Con respecto a la metodología, recordemos que se busca una segmentación en el pool de datos, donde las técnicas de agrupación como el análisis de clúster correspondientes a la minería de datos, parecen adecuados (Anderberg, 1973). Por lo tanto, el algoritmo de clústeres a desarrollar será de tipo jerárquico; el cual inicia con $\mathrm{N}$ individuos con patrones (características) individuales, calcula las distancias entre los individuos y los aglomera en grupos según criterios del vecino más cercano, más lejano o promedio (Everitt, Landau and Leese, 2001), reiteradamente, hasta consolidar en un solo grupo a todos los individuos (Xu and Wunsch, 2008). Ahora bien, en el algoritmo de clustering es necesario establecer un criterio de semejanza y agrupación; los que serán la distancia euclidiana como criterio de disimilitud y el método Ward como criterio de agrupamiento. El cual busca minimizar la varianza dentro de los grupos e iterativamente incluirá a otros integrantes, actualizando la varianza del grupo hasta terminar con todos los individuos (países) del pool de datos, a fin de que un individuo - en este caso un país - no pueda formar parte de dos grupos al mismo tiempo.

\section{RESULTADOS}

En la presente sección se presentarán los principales resultados obtenidos tras la aplicación del método de clustering en el pool de 122 países en el período de 2000 a 2017.7 Así, en la tabla 2 (ver Tabla 2) se puede identificar — según el algoritmo y las variables consideradas - una 
Tabla 2: Distribución asimétrica de países entre los clusters

\begin{tabular}{ccccccc}
\hline \multicolumn{7}{c}{ Número de países por clúster } \\
\hline Año & Clúster 1 & Clúster 2 & Clúster 3 & Clúster 4 & Clúster 5 & Total \\
\hline 2000 & 82 & 9 & 21 & 9 & 1 & 122 \\
\hline 2001 & 78 & 9 & 29 & 5 & 1 & 122 \\
\hline 2002 & 78 & 9 & 29 & 5 & 1 & 122 \\
\hline 2003 & 104 & 10 & 2 & 5 & 1 & 122 \\
\hline 2004 & 104 & 10 & 2 & 5 & 1 & 122 \\
\hline 2005 & 103 & 10 & 2 & 6 & 1 & 122 \\
\hline 2006 & 96 & 10 & 10 & 3 & 3 & 122 \\
\hline 2007 & 97 & 8 & 11 & 3 & 3 & 122 \\
\hline 2008 & 97 & 8 & 11 & 3 & 3 & 122 \\
\hline 2009 & 77 & 9 & 29 & 2 & 5 & 122 \\
\hline 2010 & 97 & 9 & 9 & 2 & 5 & 122 \\
\hline 2011 & 95 & 11 & 11 & 2 & 3 & 122 \\
\hline 2012 & 100 & 8 & 9 & 2 & 3 & 122 \\
\hline 2013 & 99 & 8 & 10 & 2 & 3 & 122 \\
\hline 2014 & 91 & 16 & 10 & 2 & 3 & 122 \\
\hline 2016 & 90 & 16 & 11 & 2 & 3 & 122 \\
\hline 2017 & 92 & 16 & 8 & 2 & 4 & 122 \\
\hline & 95 & 13 & 8 & 2 & 4 & 122 \\
\hline
\end{tabular}

Elaboración: propia.

Figura 1: Desempeño económico en los clústers

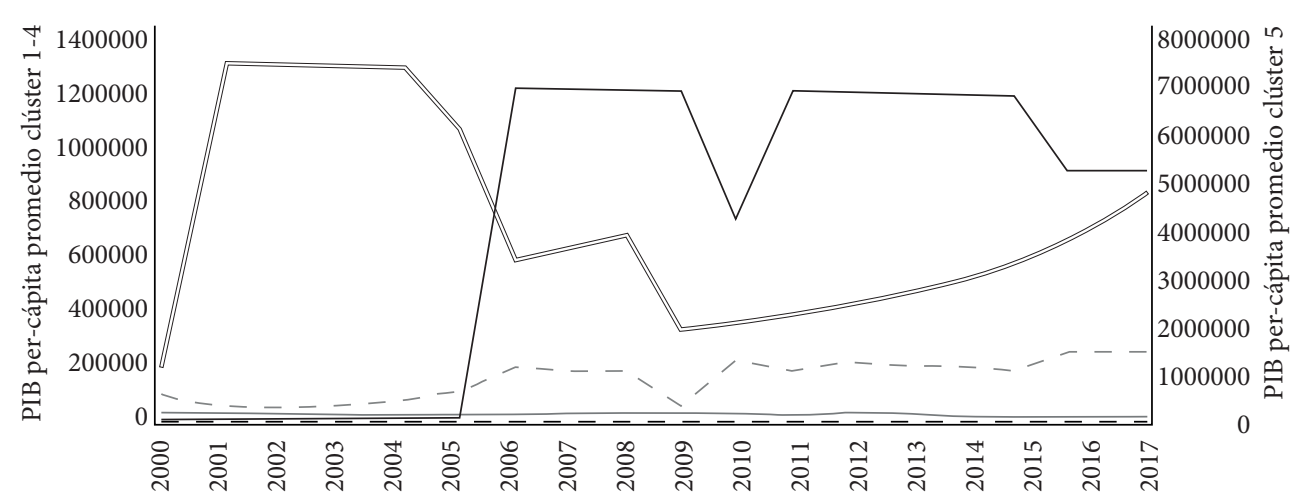

- - - Clúster 1 Clúster 2 - - Clúster $3=$ Clúster 4 Clúster 5

Elaboración: propia. 
distribución asimétrica de los países entre los cinco conglomerados. Pues en 2001 el clúster 1 abarca a 82 países, el clúster 2 a 9, el clúster 3 a 21, el clúster 4 a 9 y el clúster 5 a 1 (Estados Unidos). Donde dicha asimetría se mantiene a lo largo del período en estudio. Este hecho ya representa un primer indicio de que los países no convergen hacia un mismo estado estacionario, sino a varios. Para complementar lo anterior, en la tabla 2 se puede apreciar cómo la metamorfosis del desarrollo — según la información considerada - hace que el clúster 1 vaya aumentando su volumen - aglutinando a más países-, mientras que los clústeres 4 y 5 , al ser los de mayor desarrollo económico, se mantengan prácticamente inmutables en el tiempo y, más bien, surjan transiciones entre estos dos conglomerados - como son los casos de Indonesia, China y Corea-. Entre dichas transiciones tenemos el caso de Estados Unidos, pues la evidencia muestra que en 2000 su dinámica económica no se relaciona con ningún otro país, lo hace brevemente en el período de 2006 a 2008 junto a China y Japón, y terminar en 2017 conformando un conglomerado con China.

No obstante, la robustez del análisis de clustering no radica en una tabla de frecuencia sobre el número de individuos en cada grupo, sino en la determinación de patrones que hacen que un individuo pertenezca solo a uno de los clúster formados, de este modo la evidencia empírica recolectada en el análisis de clustering permite identificar los siguientes grupos: el primero, uno de estancamiento económico que aglutina a la mayoría de países cuyo PIB per cápita en promedio entre los países es el menos cuantioso (ver Figura 1), sugiriendo, así, que en la dinámica capitalista es necesario que ciertos países no se desarrollen o, al menos, no tanto, pues «el desarrollo de unos depende del subdesarrollo de otros» (Emmanuel, 1972); asimismo, se evidencia otro grupo se ha mantenido invariante en la economía mundial, cuya dinámica de desarrollo económico es mayor al primer clúster (ver Figura 1), pero no llega a superar sus limitaciones, manteniéndose colapsado, pues no avanza, pero tampoco decrece, por lo cual el segundo clúster será denominado del limbo. A su vez, se identifica un tercer grupo donde la mayoría - hasta 2002 - se diferenciaba del grupo de estancamiento económico, sin embargo, a partir de 2003 pasan a formar parte del primer grupo, es decir, sus economías eran muy sensibles a los vaivenes del mercado mundial y no pudieron sostenerse en el tiempo, por lo cual se le denominará al clúster 3 el grupo de la deriva; luego tenemos a los clústeres 4 y 5 , los grupos más dinámicos e inmóviles a las fuerzas del mercado, que serán denominados grupo de escalera al cielo y el edén del crecimiento, respectivamente, pues su ingreso per cápita - pese a que tiene una tendencia a la baja en el clúster 4 a partir de 2006- es superior a la de los clústeres precedentes - excepto en el período de 2000 a 2005, donde el clúster 5 es conformado únicamente por Estados Unidos, pero que aun así presenta un ingreso per cápita superior al del clúster 1 y 2 -

Así, la evidencia empírica muestra cómo la dinámica capitalista hace que el despegue prácticamente no surja apenas en unos cuantos países que han logrado salir del estancamiento económico o de un equilibrio estacionario de ingreso bajo, mientras que muchos otros se van sumando al mismo; a la vez que el crecimiento sostenido y perpetuación de países en el edén del crecimiento o equilibrio de alto ingreso promueve nuevos participantes en la hegemonía mundial de la economía, sin olvidar que muchos otros países que no se encuentran en el edén del crecimiento, escalera al cielo o estancamiento económico, pertenecen más al limbo o la deriva, 
que si bien presentan dinámicas superiores al del clúster 1, tampoco llegan a ser tan elevadas para formar parte de los conglomerados más vertiginosos.

De este modo, los resultados encontrados parecen corresponden a la literatura sobre convergencia y divergencia económica, asimismo, con los postulados dependentistas sobre el progreso y retraso en la metrópoli y satélites, respectivamente; no obstante, no son resultados aislados, propios de una u otra región, más bien responden a la estructura capitalista exacerbada en la explotación de unos en beneficios de otros, que puede ser entre países e incluso al interior de éstos, donde las sociedades difícilmente pueden llegar a un objetivo común porque los intereses individuales de agentes con poder reinan sobre agentes sin poder, al punto que bloquean los intentos de desarrollo económico para no perder su statu quo (Acemoglu y Robinson, 2014).

En adición, los gobiernos, academia y sociedad civil — entre otros- de los países en desarrollo, deberían replantear si las teorías del siglo pasado que permitieron el ascenso económico de las economías hoy conocidas como desarrolladas, serán de utilidad para el contexto actual de países subdesarrollados. Probablemente sea momento de reunir a los eruditos de cada país en desarrollo y proponer nuevas teorías ajustadas a la realidad subdesarrollada, pues como manifestó Krugman (1995), en realidad, ¿qué sabemos sobre el desarrollo económico? Quizá menos que antes y, por eso, la necesidad de plantear alternativas no solo en las agendas de crecimiento económico de cada nación, sino también y desde las aulas de clase, donde - y en gran parte a criterio propio - es difícil - pero no imposible - terminar con un sistema que se aprende desde inicio a fin en la formación académica.

Por otro lado, es importante reiterar que los resultados obtenidos corresponden a la información recopilada que, si bien es correspondiente con la literatura, deben existir otras variables que igualmente contribuyan a la explicación del desarrollo económico, pero que no han sido tomadas en cuenta, no por su pertinencia, sino más bien por su disponibilidad. Asimismo, se pretende dejar la puerta abierta para futuras investigaciones en las que se realice un análisis previos de las variables potenciales que explique el desarrollo económico como, por ejemplo, un análisis de componentes principales a fin de conocer el porcentaje de explicación con respecto al desarrollo económico, lo que a su vez puede ser complementado tras realizar dicha metodología y un análisis de clustering con una metodología de clasificación como árboles de decisión, análisis discriminante o machine learning para realizar una segmentación más profunda en el conjunto de países.

\section{CONCLUSIONES}

En el sistema capitalista parece imperante la división del mundo en desarrollados y subdesarrollados, donde la dinámica de crecimiento de los primeros, depende del atraso económico de los segundos. Un hecho que ha sido identificado por corrientes del desarrollo económico como los estructuralistas y dependentistas, que si bien proponen teorías diferentes a las escuelas de pensamiento tradicionales (dominantes) como la neoclásica, su efectividad ha sido escasa y por ello - además de otros factores - el desarrollo económico sigue enraizado en el pensamiento de países desarrollados, quienes esgrimen y funden sus políticas a su realidad, pero que difícilmente serán de utilidad para la otra cara de la moneda (los subdesarrollados). 
De hecho, la motivación del presente artículo, dada las teorías del desarrollo económico y los clubes de la convergencia en el contexto real, fue elaborar una segmentación de las economías en el período de 2000-2017, donde las dinámicas de estancamiento, limbo, deriva, escalera al cielo y el edén del crecimiento han revelado que muchos países se encuentran atorados en dichos estados, donde los extremos se asemejan a los equilibrios de bajo y alto ingreso, respectivamente, mientras otros se encuentran gravitando en las corrientes hacia el edén del crecimiento, a la vez que el grupo de estancamiento económico aumenta y el edén del crecimiento apenas mengua. De este modo, la evidencia empírica recolectada en la actual propuesta puede responder a la interrogante: ¿Las teorías sobre el desarrollo económico son efectivas para todos los países, o solo para una proporción de ellos? No, puesto que pareciera funcionar solo para una fracción en el total de países, pues la formación de clústeres, conglomerados o clubes ha sido imperante en la propuesta actual, por lo cual no todos los países pueden obtener una metamorfosis del desarrollo que les traslade de un estado estacionario de bajo ingreso a uno de alto ingreso.

\section{NOTAS}

1 Entendiendo al subdesarrollo como una fase previa al desarrollo económico según las etapas del desarrollo económico en el pensamiento de Rostow (1963).

2 En el sentido que los países subdesarrollados en su mayoría presentan balanzas comerciales deficitarias, elevado subempleo, sectores duales intensivos en manos de obra y escasa industrialización, bajo nivel de ahorro, menores tasas de crecimiento y mayores tasas de natalidad; mientras que países desarrollados exhiben gran industrialización, mayor capacidad de absorber la mano de obra excedentaria, mayor ahorro e inversión en capital, mayores tasas de crecimiento y menores tasas de natalidad (Ros, 2013; Todaro y Smith, 2012; Vera, 2001).

3 Según el nivel de ingreso per cápita estimado por el método Atlas, la clasificación es: países de ingreso bajo, con un ingreso de USD 995 o menos; países de ingreso mediano bajo, cuyo ingreso oscila entre USD 996 y USD 3895; países de ingreso mediano alto, con ingreso entre USD 3896 y USD 12.055; y países de ingreso alto, con ingresos igual o mayores a USD 12.056, según Todaro y Smith (2012).

4 Los estudios mencionados sobre análisis del proceso de convergencia en la Unión Europea fueron realizados en el espíritu del algoritmo de clustering diseñado por Phillips y Sul (2007).

5 Fuerzas gravitatorias en el sentido de que atraen nuevamente hacia el estado estacionario de bajo ingreso. 6 Para una revisión de cada uno de los criterios de selección óptimo, así como del algoritmo que se encuentra detrás del análisis de clúster, se puede revisar la documentación del paquete NbClust, disponible en https://www.rdocumentation.org/packages/NbClust/versions/3.o/topics/NbClust.

7 Ya que el enfoque de estudio albergó un pool de países tan grande, incluir todos los cuadros y el detalle de los países por clúster excede los espacios y normas permitidas por la presente revista, por ello, en el siguiente link se puede encontrar a profundidad el detalle sobre los países y su transición en el período de estudio: https://drive.google.com/open?id=11pH4_z_Lmza6-BHWELBV6-BtocmCLXLn

\section{REFERENCIAS}

Amin, S. (1974). La acumulación a escala mundial. México DF, México: Siglo XxI.

Amin, S., Bettelheim, C., Emmanuel, A. y Palloix, Ch. (1976). Imperialismo y comercio internacional (El intercambio desigual). México DF, México: Cuadernos Pasado y Presente.

Acemoglu, D. y Robinson, J. (2014). Por qué fracasan los países. Los orígenes del poder, la prosperidad y la pobreza. Madrid, España: Deusto Ediciones. 
Anderberg, M. (1973). Cluster analysis for applications. London, UK: Academic Press.

Baddeley, M. (2006). Convergence or Divergence? The impacts of globalisation on growth and inequality in less developed countries. International Review of Applied Economics, $20(3), 391-410$.

Baer, W. (1963). La economía de Prebisch y de la CEPAL, El Trimestre Económico, 30(117), 144-161.

Ball, G. and Hall, D. (1965). Isodata, a novel method of data analysis and pattern classification. Stanford Research Institute. Recuperado de https://apps.dtic.mil/dtic/tr/fulltext/u2/699616.pdf.

Baran, P. (1950). On the political economy of backwardness. Reprinted in Agarwala \& Singh.

Barro, R. and Salai-Martin, X. (1995). Growth models with poverty traps. Economic Growth. Cambridge, USA: MIT Press, MA.

Ben-David, D. (1998). Convergence clubs and subsistence economies. Journal of Development Economics, 55(1), 155-177.

Borsi, M. and Metiu, N. (2015). The evolution of economic convergence in the European Union. Empirical Economics, 48(2), 657-681.

Brida, J., Garrido, N. and Muredu, F. (2014). Italian economic dualism and convergence clubs at regional level. Quality \& Quantity, 48(1), 439-456.

Botta, A. (2010). Economic development, structural change and natural resource booms: a structuralist perspective. Metroeconomica, 61(3).

Chatterji, M. (1992). Convergence clubs and endogenous growth. Oxford Review of Economic Policy, $8(4), 57-69$.

De Siano, R. y D'Uva, M. (2006). Club convergence in European regions. Applied Economics Letters, 13(9), 569-574.

Dixon, R. and A. Thirlwall. (1975) A model of regional growth rate differences on Kaldorian Lines, Oxford Economic Papers.

Dutt, P., Mitra, D. and Rajan, P. (2009). International trade and unemployment: theory and cross national evidence. Journal of International Economics.

Easterly, W. (2006). The big push déjà vu: a review of Jeffrey Sachs's the end of poverty: economic possibilities for our time. Journal of economic literature, 44(1), 96-105.

Emmanuel, A. (1972). Unequal exchange: a study in the imperialism of trade. New York, UsA: Monthly Review Press.

Evans, P. (1998). Using panel data to evaluate growth theories. International Economic Review, 39(2), 295-306.

Everitt, B. S., Landau, S. and Leese, M. (2001). Cluster analysis. John Wiley and Sons, Inc.

Frey, D., Frey, M. and Wieslhuber, C. (2013). Do natural resources define convergence clubs? Empirical evidence from the Kazakh regions. Economic Systems, 37(3), 404-414.

Gunder Frank, A. (1969). The development of underdevelopment. In Latin America: underdevelopment or revolution. New York, USA: Monthly Review Press.

Hartigan, J. (1975). Clustering algorithms. New York, UsA: Wiley.

Hubert, L. and Levin, J. (1976). A general statistical framework for assessing categorical clustering in free recall. Psychological Bulletin, 83(6), 1072-1080.

Krzanowski, W. and Lai, Y. (1988). A criterion for determining the number of groups in a data set using sum-of-squares clustering. Biometrics, (44), 23-34.

Krueger, A. (1990). Fallas del gobierno en el desarrollo, Criterio Jurídico, 12(1). (s. d.). 
Krugman, P. (1995). The fall and rise of development economics, in development, geography and economic theory. Cambridge, usa: MIT Press, ma.

Maasoumi, E., Racine, J. and Stengos, T. (2007). Growth and convergence: a profile of distribution dynamics and mobility. Journal of Econometrics, 136(2), 483-508.

Marini, R. M. (1974). Dialéctica de la dependencia. México DF, México: Ediciones Er.

McCoskey, S. (2002). Convergence in Sub-Saharan Africa: a nonstationary panel data approach. Applied Economics, 34(7), 819-829.

Meier, G. (2001) La Vieja Generación de Economistas del Desarrollo y la Nueva. En G. M. Meier y J. Stiglitz (Eds.), Fronteras de la economía del desarrollo; el futuro en perspectiva. Washington, D.C., USA: Banco Internacional de Reconstrucción y Fomento / Banco Mundial

Milligan, G. (1980). An examination of the effect of six types of error perturbation on fiftenn clustering algorithms. Psychometrika, 45(3), 325-342.

Murphy, K., Shleifer, A. and Vishny, R. (1989). Industrialization and the big push. Journal of Political Economy, (97), 1003-1026.

Myrdal, G. (1957). Teoría económica y regiones subdesarrolladas. México DF, México: Fondo de Cultura Económica.

Ocampo, J. A. (2001). Raúl Prebisch y la Agenda del Desarrollo en los albores del siglo xxi, Revista de la CEPAL, (75), 25-40.

Palma, G. (1978). Dependency and development: a critical overview. World Development. (s. d.).

Prebisch, Raúl. (1950). The economic development of Latin America and its principal problems. New York, UsA: United Nations.

Postiglione, P., Benedetti, R. and Lafratta, G. (2010). A regression tree algorithm for the identification of convergence clubs. Computational Statistics \& Data Analysis, 54(11), 2776-2785.

Quah, D. (1996). Twin peaks: growth and convergence in models of distribution dynamics. The Economic Journal, 106(437), 1045-1055.

Ratkowsky, D. and Lance, G. (1978). A criterion for determining the number of groups in a classification. Austalian Computer Journal, (10), 115-11\%.

Romaguera, P. (1992). Restricciones al crecimiento: aplicación de un modelo de brechas a la economía chilena, Coleccion Estudios Cieplan, (36), 145-182.

Ros, J. (2013). Stylized Facts. Rethinking Economic Development, Growth and Institutions. Oxford, UK: OUP.

Rostow, Walt W. (1963). The take-off into self-sustained growth. En Barry E. Supple (ed.), The experience of economic growth: case studies in economic history, New York, UsA: Random House.

Rousseeuw, P. (1987). Silhouettes: a graphical aid to the interpretation and validation of cluster analysis. Journal of Computational and Applied Mathematics, (20), 53-65.

Sarkar, P. (1997). Growth and terms of trade: a North-South Macroeconomic framework, Journal of Macroeconomics, 19(1), 117-133.

Snowdown, B. (2009). The Solow Model, poverty traps, and the foreign aid debate. History of Political Economy, 41(1), 241-262.

Solow, R. (1956). A contribution to the theory of economic growth. The Quarterly Journal of Economics, $70(1), 65-94$.

Swan, T. (1956). Economic growth and capital accumulation. Economic record, 32(2). 
Thirlwall, A. (1983). Foreign Trade elasticities in centre-periphery models of growth and development, PSL Quarterly Review, 36(146), 249-261.

Todaro, M. and S. Smith (2012) Comparative economic development. In Addison-Wesley, Economic Development. Boston, UsA.

Ward, J. (1963), Hierarchical grouping to optimize an objective function. Journal of the American Statistical Association, (58), 236-244.

$\mathrm{Xu}, \mathrm{R}$. and Wunsch, D. (2008). Clustering. New Jersey, UsA: John Wiley \& Sons, Inc. 